\title{
1-year outcomes for lung transplantation recipients with non-alcoholic fatty liver disease
}

\author{
Anil J. Trindade ${ }^{1,2}$, Tany Thaniyavarn ${ }^{1}$, Nikroo Hashemi ${ }^{3}$, Antonio Coppolino III $^{4}$, John C. Kennedy ${ }^{1}$, \\ Hari R. Mallidi ${ }^{4,5}$, Souheil El-Chemaly ${ }^{1}$ and Hilary J. Goldberg ${ }^{1}$
}

${ }^{1}$ Division of Pulmonary and Critical Care Medicine, Brigham and Women's Hospital/Harvard Medical School, Boston, MA, USA. ${ }^{2}$ Division of Allergy, Pulmonary and Critical Care Medicine, Vanderbilt University Medical Center, Nashville, TN, USA. ${ }^{3}$ Division of Gastroenterology, Hepatology and Endoscopy, Brigham and Women's Hospital and Harvard Medical School, Boston, MA, USA. ${ }^{4}$ Division of Thoracic Surgery, Brigham and Women's Hospital/Harvard Medical School, Boston, MA, USA. ${ }^{5}$ Division of Cardiac Surgery, Brigham and Women's Hospital/Harvard Medical School, Boston, MA, USA.

Corresponding author: Hilary J. Goldberg (hjgoldberg@bwh.harvard.edu)

Shareable abstract (@ERSpublications)

In this single-centre, retrospective analysis of lung transplant recipients, we identified that mildmoderate non-alcoholic fatty liver disease is associated with acceptable perioperative and 1-year outcomes https://bit.ly/36WNzhi

Cite this article as: Trindade AJ, Thaniyavarn T, Hashemi N, et al. 1-year outcomes for lung transplantation recipients with non-alcoholic fatty liver disease. ERJ Open Res 2021; 7: 00103-2021 [DOI: 10.1183/23120541.00103-2021].

Copyright (The authors 2021

This version is distributed under the terms of the Creative Commons Attribution NonCommercial Licence 4.0. For commercial reproduction rights and permissions contact permissions@ersnet.org

Received: 10 Feb 2021 Accepted: 13 May 2021

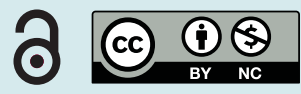

\section{Abstract}

Advanced hepatic fibrosis and cirrhosis are absolute contraindications to lung transplantation. [1] However, whether fatty liver disease with mild-moderate fibrosis contributes to increased adverse outcomes postlung transplantation remains unknown.

We present a retrospective analysis of patients transplanted at Brigham and Women's Hospital between 2015 and 2017 to identify whether patients with mild-moderate non-alcoholic fatty liver disease (NAFLD) experience increased short-term complications compared to patients with normal liver architecture. Patients with advanced (F3-F4) fibrosis and/or cirrhosis were considered non-suitable transplant candidates, a priori. This study was powered for a difference in index hospital-free days within the first 30 days of $25 \%(\alpha=0.05, \beta=0.8)$. Secondary outcomes included index intensive care unit (ICU)-free days within the first 10 days post-transplant, perioperative blood product transfusion, incidence of index hospitalisation arrhythmias and delirium, need for insulin on discharge post-transplant, tacrolimus dose required to maintain a trough of 8-12 ng. $\mathrm{mL}^{-1}$ at index hospital discharge, and 1-year post-transplant incidence of insulin-dependent diabetes, acute kidney injury, acute cellular rejection, unplanned hospital readmissions and infection.

150 patients underwent lung transplantation between 2015 and 2017 and were included in the analysis; of these patients 40 (27\%) had evidence of NAFLD. Median index hospital-free days for patients with NAFLD were non-inferior to those without (16 days, IQR 10.5-19.5 versus 12 days, IQR 0-18.0, p=0.03). Regarding secondary outcomes, both index hospitalisation and 1-year outcomes were non-inferior between patients with NAFLD and those with normal liver architecture.

This study demonstrates that mild-moderate severity NAFLD may not be a contraindication to lung transplantation.

\section{Introduction}

Beginning in 2015, lung transplant candidates at Brigham and Women’s Hospital (BWH) with risk factors for liver disease underwent in-depth, stepwise evaluation to better characterise and risk-stratify patients for severe liver disease. This protocol was instituted after experiencing several episodes of fulminant hepatic failure post-transplant in patients without previously known liver disease based on standard serologic assessment of liver function tests, albumin and coagulation studies. The intent of the new protocol was to obtain a basic assessment of liver architecture in all lung transplant candidates via ultrasonography, while more carefully screening for liver abnormalities in patients with known risk factors. Our protocol identified 
a large percentage of patients with mild-moderate liver disease. While cirrhosis and advanced hepatic fibrosis are accepted contraindications to lung transplantation, whether mild-moderate fatty liver disease contributes to untoward outcomes post-lung transplantation is unknown [1]. This question is of increasing importance given the wide prevalence of non-alcoholic fatty liver disease (NAFLD) in the general population. NAFLD currently afflicts $20-40 \%$ of the worldwide population [2]. NAFLD, defined as replacement of $>5 \%$ of the liver with fat in the absence of other causes of hepatic inflammation (viral disease, $>20 \mathrm{~g}$ alcohol use per day, drug toxicity and autoimmune disease), is a risk factor for non-alcoholic steatohepatitis (NASH), fibrosis, cirrhosis and hepatocellular carcinoma [2].

NAFLD is also associated with a multitude of extrahepatic comorbidities, including hypertension, hyperlipidaemia, diabetes, atrial fibrillation, coronary artery disease, venous thromboembolism, infection, weakness and chronic kidney disease [2, 3-8]. NAFLD is more than just correlative to these comorbidities; rather, evidence suggests that the upregulated proinflammatory process induced by NAFLD is causative. For example, NAFLD promotes insulin resistance by disrupting long-chain fatty acid digestion and creating intermediates that downregulate metabolic pathways such as Akt/mTORC2 [2, 9, 10]. Moreover, NAFLD causes an accelerated decline in renal function by augmenting intrarenal proinflammatory pathways including connective tissue, epidermal and platelet-derived growth factors (CTGF, EGGF, PDGF) to promote glomerulosclerosis and tubular atrophy [10, 11]. NAFLD contributes to vascular disease by upregulating oxidative stress pathways, with subsequent upregulation of tumour necrosis factor- $\alpha$ and interleukin-6 pathways, activation of coagulation cascades, inhibition of fibrinolysis, increased vascular tone and resultant intimal thickening [10-12]. Finally, NAFLD can promote sarcopenia via insulin resistance and resultant myosteatosis, protein catabolism and myostatin-induced proteolysis [13].

Lung transplantation, and the related need for immunosuppression, is also associated with increased morbidity; allograft recipients have an increased incidence of diabetes, hypertension, hyperlipidaemia, atrial arrhythmias, skeletal muscle weakness and chronic kidney disease [14-17]. Whether the complications of lung transplantation are additive to, or perhaps synergistic with, that of the proinflammatory effects of NAFLD is unknown.

Given the association of hepatic steatosis with a proinflammatory state and its subsequent effect on cardiovascular disease, insulin resistance, kidney injury and infection, we hypothesised that lung allograft recipients with mild-moderate NAFLD would incur greater complications within the first-year post-transplantation. The primary outcome of this study is index hospital-free days within the first 30 days. Secondary outcomes include index intensive care unit (ICU)-free days, perioperative blood product transfusion, incidence of index hospitalisation arrhythmias and delirium, need for insulin on discharge post-transplant and tacrolimus dose required to maintain a goal trough of $8-12 \mathrm{ng} \cdot \mathrm{mL}^{-1}$ at hospital discharge post-transplant. Other secondary outcomes include incidence of insulin-dependent diabetes, acute kidney injury, acute cellular rejection, unplanned hospital readmissions and infection, all at 1 year post-transplant.

\section{Methods}

Permission to perform this retrospective analysis was obtained from the institutional review board at BWH prior to collecting data from the electronic record. Patients aged >18 years who underwent lung transplantation evaluation at BWH between January 1, 2015 and December 31, 2017 were included; a total of 150 patients met inclusion criteria and were transplanted (figure 1). As part of the routine assessment, all patients underwent a screening abdominal ultrasound. Patients with risk factors for hepatic dysfunction underwent more detailed assessment of liver fibrosis (see figures 2 and 3). We decided $a$ priori that severe fibrosis (F3-F4) and cirrhosis were absolute contraindications to lung transplantation. Post-lung transplantation care at BWH was per the usual protocol and has been previously published [18].

\section{Diagnosis of NAFLD}

Abdominal ultrasound was performed in all lung transplant candidates, per our standard evaluation protocol. In patients with risk factors for hepatic disease (see figure 2), we performed an additional diagnostic algorithm. Our hepatology colleagues provided guidance regarding which additional series of noninvasive testing was necessary to exclude significant fibrosis/cirrhosis in order to obviate the need for liver biopsy (figure 3). Selected noninvasive diagnostic studies included hepatic transient elastography (FibroScan ${ }^{\circledR}$, Echosens SA, Paris, France), serum biomarker profiling (FibroSure, LabCorp, Burlington, NC, USA) and cross-sectional imaging (with computed tomography (CT) or magnetic resonance imaging (MRI)). The evaluation to exclude advanced NAFLD is based upon pragmatic expert opinion, as even within the hepatology field, "a validated, widely accepted procedure for the diagnosis and monitoring of NAFLD does not yet exist” [19]. Nevertheless, noninvasive studies are useful for excluding advanced 
Patients referred for lung transplantation evaluation at Brigham and

Women's Hospital between January 1, 2015 and December 31, 2017 $(n=500)$

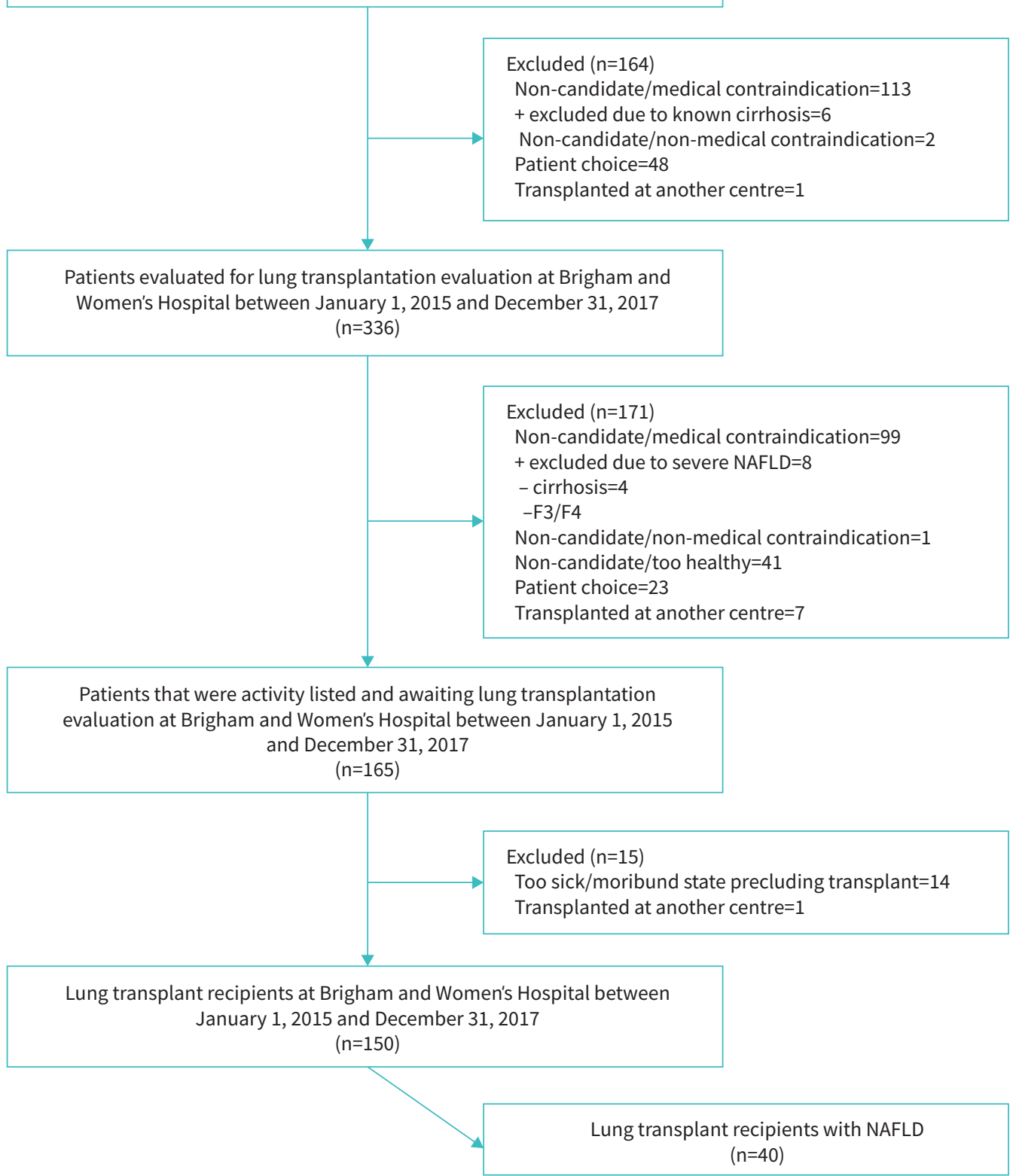

FIGURE 1 Flow chart of patients included in the analysis. NAFLD: non-alcoholic fatty liver disease.

disease (F3 or F4 disease on the Metavir scoring system) [20-22]. For example, ultrasound has a sensitivity $\sim 85 \%$ and specificity $\sim 95 \%$ to detect moderate steatosis [21]. Hepatic transient elastography (FibroScan ${ }^{\circledR}$ ), which utilises applied mechanical excitation to detect tissue elasticity, is well validated in patients with chronic hepatitis B and C, alcoholic liver disease and NAFLD, with a negative predictive value of $97 \%$ for advanced fibrosis [19, 23-26]. Hepatic transient elastography (FibroScan ${ }^{\circledR}$ ) results were correlated to histologic Metavir scores as follows: <2 kPa=F0, 2-7 kPa=F0-F1, 7.5-10 kPa=F2, 10-14 kPa=F3, $>14 \mathrm{kPa}=\mathrm{F} 4$ [26]. The FibroSure test is a panel of six serologic markers (ALT, GGT, total bilirubin, haptoglobin, apolipoprotein A1 and $\alpha-2$ microglobulin) to assess liver scarring and correlates with biopsy $\sim 75 \%$ of the time in patients with hepatitis B, hepatitis C, alcoholic liver disease and NAFLD [27-29]. FibroSure results were classified as $0-0.21=\mathrm{F} 0,0.21-0.27=\mathrm{F} 0-\mathrm{F} 1,0.27-0.31=\mathrm{F} 1,0.31-0.48=\mathrm{F} 1-\mathrm{F} 2,0.48-$ $0.58=\mathrm{F} 2,0.58-0.72=\mathrm{F} 3,0.72-0.74=\mathrm{F} 3-\mathrm{F} 4,>0.75=\mathrm{F} 4$ [29]. In cases of discordance between FibroScan and FibroSure results, or where a diagnosis of advanced fibrosis was equivocal, cross-sectional imaging and/or 
a) Known liver disease, including non-alcoholic fatty liver disease

b) Abnormal liver function tests

c) Abnormal liver imaging on screening abdominal ultrasonography

d) Systemic disease that can potentially involve the liver

e) Known telomeropathy

f) Exposure to hepatitis B or hepatitis C

g) Chronic or heavy alcohol used ( $>7$ drinks/week for females, $>14$ drinks/week for males)

h) Other conditions that predispose to hepatopathy (e.g. right heart failure)

FIGURE 2 Indications to undergo protocolised evaluation of liver function.

liver biopsy were used as the arbiter of hepatic fibrosis severity. Notably, multidetector CT can detect $\geqslant$ F2 with a sensitivity of $70-80 \%$ and a specificity $~ 85 \%$ [30-32]. While magnetic resonance imaging is the preferred mode of cross-sectional imaging, it is not always feasible in patients with end-stage lung disease who have marginal gas-exchange [32, 33].

\section{Statistical analysis}

The sample size of this retrospective analysis was calculated with regard to a primary outcome of index hospital-free days within the initial 30 days post-transplant. We performed a power calculation based on the median hospital length of stay in our cohort of 18 days (IQR 11-25). We identified a sample size of 150 subjects to detect a $25 \%$ increase in hospital-free days in the NAFLD cohort, with $\alpha=0.05$ and $\beta=0.2$, understanding that the rate of NAFLD in the general population is $\sim 20 \%$.

Statistical analysis was performed using STATA version 15. 1 (StatCorp LLC, College Station, TX, USA). For all results, p-values $\leqslant 0.05$ were considered significant. Differences in baseline demographic data were assessed using Fisher's exact test for binary data, Pearson's chi-square test for categorical variables and Wilcoxon's rank sum tests for continuous data, as none of the continuous variables were normally distributed.

We performed univariate analyses using Fisher's exact test to assess for a relationship between NAFLD and binary outcomes; continuous variables were dichotomised above and below the median for these analyses. Multivariate logistic regression analyses were then performed, including covariates that were significant on univariate assessment at $\mathrm{p}<0.1$.

\section{Definitions}

We utilised the following definitions when identifying outcomes: 1) delirium = critical care nursing diagnosis and documentation using the Confusion Assessment Method for the Intensive Care Unit (CAM-ICU) clinical tool and/or administration of therapy for agitation [34]; 2) hypertension = need for left ventricular afterload-reducing medications in the post-transplant setting; 3) diabetes = need for insulin therapy (haemoglobin A1c\% is not routinely checked in the tertiary setting at BWH); 4) acute cellular rejection = any pathologic finding of $\geqslant \mathrm{A} 1$ or $\geqslant \mathrm{B} 1 \mathrm{R}$ rejection by the International Society for Heart and Lung Transplantation (ISHLT) criteria [35]; 5) infection = suspected or documented infection necessitating

a) Assessment of hepatitis $B$ and hepatitis $C$ serologies and abdominal ultrasound in all candidates

b) In patients with increased risk factors for hepatic disease: FibroSure score AND/OR Hepatic transient elastography (FibroScan)

c) Confirmatory cross-sectional liver imaging (with CT and MRI), as needed

d) Hepatology consultation for consideration of liver biopsy if liver disease suspected despite a negative noninvasive evaluation

FIGURE 3 Evaluation of liver architecture in lung transplant candidates. CT: computed tomography; MRI: magnetic resonance imaging. 
hospitalisation; and 6) kidney function was estimated using the Chronic Kidney Disease Epidemiology Collaboration (CKD-EPI) formula for glomerular filtration rate [36].

Results

Patient recruitment

During the inclusion period, 500 patients were referred to BWH for lung transplantation consideration, of which 336 patients underwent the formal evaluation process. In this cohort 232 patients (69\%) met criteria for undergoing more detailed evaluation for hepatic disease (figures 2 and 3). Twenty-one patients (9\%) required liver biopsy. Notably, 8 out of 232 patients (3.4\%) were deemed non-viable lung transplant candidates due to F3/F4 fibrosis or cirrhosis. Six out of the eight patients were diagnosed with advanced fibrosis via liver biopsy and the other two with cross-sectional imaging (CT and MRI, respectively; see figure 1). Ultimately 150 patients underwent lung transplantation at BWH during the 2015-2017 time period, of which 40 patients had mild-moderate NAFLD.

\section{NAFLD diagnosis}

NAFLD was identified in 40 out of 150 (27\%) of our cohort by one or more screening modalities. Abdominal ultrasound detected abnormal liver architecture in 35 out of 40 patients in our cohort with NAFLD (sensitivity $=87.5 \%$ ). The remaining five patients with normal abdominal ultrasound results were diagnosed via FibroScan and/or FibroSure testing; these patients underwent advanced testing due to short telomere syndrome, history of abnormal transaminases on serologic testing, history of hepatitis C and history of alcohol abuse. Nine of the 40 patients (23\%) with NAFLD had steatosis without fibrosis, as diagnosed by ultrasound, FibroScan, FibroSure cross-sectional imaging and/or liver biopsy. Thirty-one (77.5\%) patients who were ultimately diagnosed with NAFLD underwent transient elastography (FibroScan) testing. FibroScan was non-diagnostic in 3 out of 31 patients (9.7\%). FibroScan testing showed F0-F1, F2, F3 and F4 hepatic fibrosis in 24 (60\%), 2 (5\%), 1 (2.5\%) and 1 (2.5\%) patient(s), respectively, among the NAFLD cohort. The two patients diagnosed with F3 and F4 disease via FibroScan underwent liver biopsies that were not suggestive of advanced fibrosis and were ultimately transplanted. FibroSure testing was performed in 17 out of 40 patients and was non-diagnostic in one of those patients (5.8\%). FibroSure testing showed F0, F1-F2, F2, F3 and F4 disease in 6, 5, 3, 1 and 1 patient(s), respectively. The two patients with advanced fibrosis underwent cross-sectional imaging and/or liver biopsy, which were not felt to be consistent with advanced fibrosis (see table 1), and the patients were ultimately transplanted. Eleven of the 40 patients underwent both FibroScan and FibroSure testing. The results were concordant in eight patients, with each test showing $\geqslant$ F2 fibrosis; this is similar to findings in other patient populations, such as those with hepatitis $C$ virus [37]. In two of the three cases where FibroScan and FibroSure did not agree, liver biopsy was performed, and results were more concordant with FibroScan findings. CT imaging was performed in 10 patients, 8 of whom had FibroScan or FibroSure testing; CT imaging was performed in instances where patients could not tolerate MRI due to the severity of hypoxaemia. CT imaging was concordant with FibroSure and FibroScan results in 9/10 instances.

\section{Baseline characteristics}

NAFLD was significantly associated with body mass index (median and interquartile range body mass index (BMI) for patients with NAFLD 29.1 (26.7-31.1) versus 25.5 (22.7-28.9) for those without, $\mathrm{p}=0.0004)$ and end-stage lung disease diagnosis, with a higher rate of interstitial lung disease in patients with NAFLD (see table 2, $\mathrm{p}=0.019$ ). There was no correlation between NAFLD and lung allocation score, 6-min walk distance or allograft ischaemic time (see table 2). There was a trend towards significant association between NAFLD and pre-transplant incidence of hyperlipidaemia ( $53 \%$ versus 35\%, $\mathrm{p}=0.059$ ) and age (63.4 (55.7-66.9) versus 58.8 (48.9-65.9) years, $\mathrm{p}=0.062)$.

\section{Univariate analysis}

NAFLD had no adverse effect on post-transplant outcomes, as seen on univariate analyses (table 3). There was no significant difference in blood product transfusion rate, delirium, requisite tacrolimus dosing, diabetes, atrial fibrillation, venous thromboembolism, acute cellular rejection, infectious complications requiring hospitalisation or incidence of cytomegalovirus (CMV) viraemia observed between groups. Of the 6 patients with NAFLD that developed CMV viraemia post-transplant, only 2 were of high-risk serostatus (CMV $\mathrm{D}^{+} / \mathrm{R}^{-}$); in patients without NAFLD, 7 of the 19 instances of CMV viraemia were in patients that were CMV mismatched. Interestingly, NAFLD was associated with improved number of hospital-free days within the first 30 days following transplantation (median 16 days, IQR 10.5-19.5 versus median 12 days, IQR 0-18.0, p=0.03). Finally, at 1 year post-transplant, patients with NAFLD had greater preservation in renal function; the median decline in baseline GFR 
TABLE 1 Identification and distribution of hepatic fibrosis grade among transplant recipients with non-alcoholic fatty liver disease

\begin{tabular}{|c|c|c|c|c|c|c|c|c|c|}
\hline Patient & $\begin{array}{l}\text { Screening } \\
\text { indication }\end{array}$ & $\begin{array}{c}\text { US } \\
\text { abdomen } \\
\text { result }\end{array}$ & $\begin{array}{l}\text { FibroScan } \\
\text { result } \mathrm{kPa}\end{array}$ & $\begin{array}{c}\text { FibroScan } \\
\text { fibrosis } \\
\text { grade }\end{array}$ & $\begin{array}{l}\text { FibroSure } \\
\text { result }\end{array}$ & $\begin{array}{c}\text { FibroSure } \\
\text { fibrosis } \\
\text { grade }\end{array}$ & $\begin{array}{l}\mathrm{CT} \text { abdomen } \\
\text { result }\end{array}$ & $\begin{array}{c}\text { MRI } \\
\text { abdomen } \\
\text { result }\end{array}$ & $\begin{array}{l}\text { Liver biopsy } \\
\text { result }\end{array}$ \\
\hline 1 & ABN US & Steatosis & - & - & - & - & Steatosis & - & - \\
\hline 2 & ABN US & Steatosis & - & - & - & - & - & Steatosis & - \\
\hline 3 & ABN US & Steatosis & - & - & - & - & Mild steatosis & - & - \\
\hline 4 & ABN US & Steatosis & 5.1 & F0-F1 & 0.52 & F2 & - & - & Steatosis \\
\hline 5 & ABN US & Steatosis & 4.2 & F0-F1 & 0.54 & F2 & - & - & - \\
\hline 6 & Short telomere & Normal & 4.9 & F0-F1 & - & - & - & - & - \\
\hline 7 & ABN US & Steatosis & 6.9 & F0-F1 & 0.33 & F1-F2 & - & - & - \\
\hline 8 & ABN US & Steatosis & 3.9 & F0-F1 & NR & - & - & - & - \\
\hline 9 & ABN US & Steatosis & - & - & 0.54 & F2 & - & - & - \\
\hline 10 & ABN US & Steatosis & - & - & - & - & - & - & - \\
\hline 11 & ABN US & Steatosis & 5.4 & F0-F1 & - & - & Normal & - & - \\
\hline 12 & ABN US & Steatosis & - & - & 0.14 & F0 & - & - & - \\
\hline 13 & ABN US & Steatosis & - & - & 0.32 & F1-F2 & Normal & - & - \\
\hline 14 & ABN US & Steatosis & 4.9 & F0-F1 & - & - & - & - & - \\
\hline 15 & $\begin{array}{l}\text { Short telomere/ } \\
\text { ethanol abuse }\end{array}$ & Normal & 5.5 & F0-F1 & - & - & Normal & - & - \\
\hline 16 & ABN US & Steatosis & 4.4 & F0-F1 & - & - & - & - & - \\
\hline 17 & ABN US & Steatosis & NR & - & 0.12 & Fo & - & - & - \\
\hline 18 & ABN US & Steatosis & 7.1 & F1 & 0.18 & F0 & Normal & - & - \\
\hline 19 & ABN LFTs & Normal & 44.3 & F4 & 0.69 & F3 & - & Normal & Mild fibrosis \\
\hline 20 & ABN LFTs & Normal & 5.1 & F0-F1 & - & - & - & - & - \\
\hline 21 & ABN US & Steatosis & 6.2 & F0-F1 & - & - & - & - & - \\
\hline 22 & ABN US & Steatosis & 7.7 & F2 & - & - & - & - & - \\
\hline 23 & ABN US & Steatosis & 6.1 & F0-F1 & - & - & Normal & - & - \\
\hline 24 & $\mathrm{HCV}$ & Normal & NR & - & 0.86 & $\mathrm{~F} 4$ & & Normal & - \\
\hline 25 & ABN US & Steatosis & 4.8 & F0-F1 & 0.19 & F0 & Normal & - & - \\
\hline 26 & ABN US & Steatosis & 7 & F0-F1 & 0.39 & F1-F2 & - & - & - \\
\hline 27 & ABN US & Steatosis & 5.4 & F0-F1 & - & - & - & - & - \\
\hline 28 & ABN US & Steatosis & 4.9 & F0-F1 & - & - & - & - & - \\
\hline 29 & ABN US & Steatosis & 2.3 & F0-F1 & - & - & - & - & - \\
\hline 30 & ABN US & Steatosis & - & - & - & - & - & Steatosis & - \\
\hline 31 & ABN US & Steatosis & 6.8 & F0-F1 & - & - & - & - & - \\
\hline 32 & ABN US & Steatosis & 4.2 & F0-F1 & - & - & - & - & - \\
\hline 33 & ABN US & Steatosis & 4.5 & F0-F1 & - & - & - & - & - \\
\hline 34 & ABN US & Steatosis & - & - & - & - & - & Steatosis & - \\
\hline 35 & ABN US & Steatosis & 3.5 & F0-F1 & - & - & - & - & - \\
\hline 36 & ABN US & Steatosis & NR & - & - & - & - & - & Mild steatosis \\
\hline 37 & ABN US & Steatosis & 10.1 & F3 & 0.1 & F0 & Normal & - & Mild fibrosis \\
\hline 38 & ABN US & Steatosis & 6.8 & F0-F1 & 0.47 & F1-F2 & Normal & - & - \\
\hline 39 & ABN US & Steatosis & 3.6 & F0-F1 & 0.17 & F0 & Normal & - & - \\
\hline 40 & ABN US & Steatosis & 4.4 & F0-F1 & 0.46 & F1-F2 & Normal & - & - \\
\hline
\end{tabular}

CT: computed tomography; MRI: magnetic resonance imaging; ABN US: abnormal ultrasound; ABN LFTs: abnormal liver function tests; $\mathrm{HCV}$ : hepatitis C virus.

was 48\% (IQR 33-57\%) for patients with NAFLD versus 58\% (IQR 42-68\%) for those without (CKD-EPI formula, $\mathrm{p}=0.03$ ).

Multivariate logistic regression analysis

We adjusted for age, BMI, presence of pre-transplant hyperlipidaemia and lung allocation score disease diagnosis group, as these were all found to be significantly associated with NAFLD at a $\mathrm{p}<0.10$. We evaluated outcomes that were either significant on univariate analysis or were clinically relevant. On multivariate analysis, there was a trend towards increased need for platelet transfusion during the peri-transplant surgery period in patients with NAFLD $(\mathrm{OR}=2.23, \mathrm{p}=0.06)$; there were no associations between NAFLD and other forms of blood product transfusion needs. Patients with NAFLD experienced increased hospital-free days within the first 30 days post-transplant (OR= 3.34, 95\% CI 0.50-6.19, $\mathrm{p}=0.02$ ). The relationship between NAFLD and improved renal function seen on univariate analysis was no longer significant after adjusting for the above confounders. See table 4 for further details. 
TABLE 2 Baseline demographics of transplant recipients

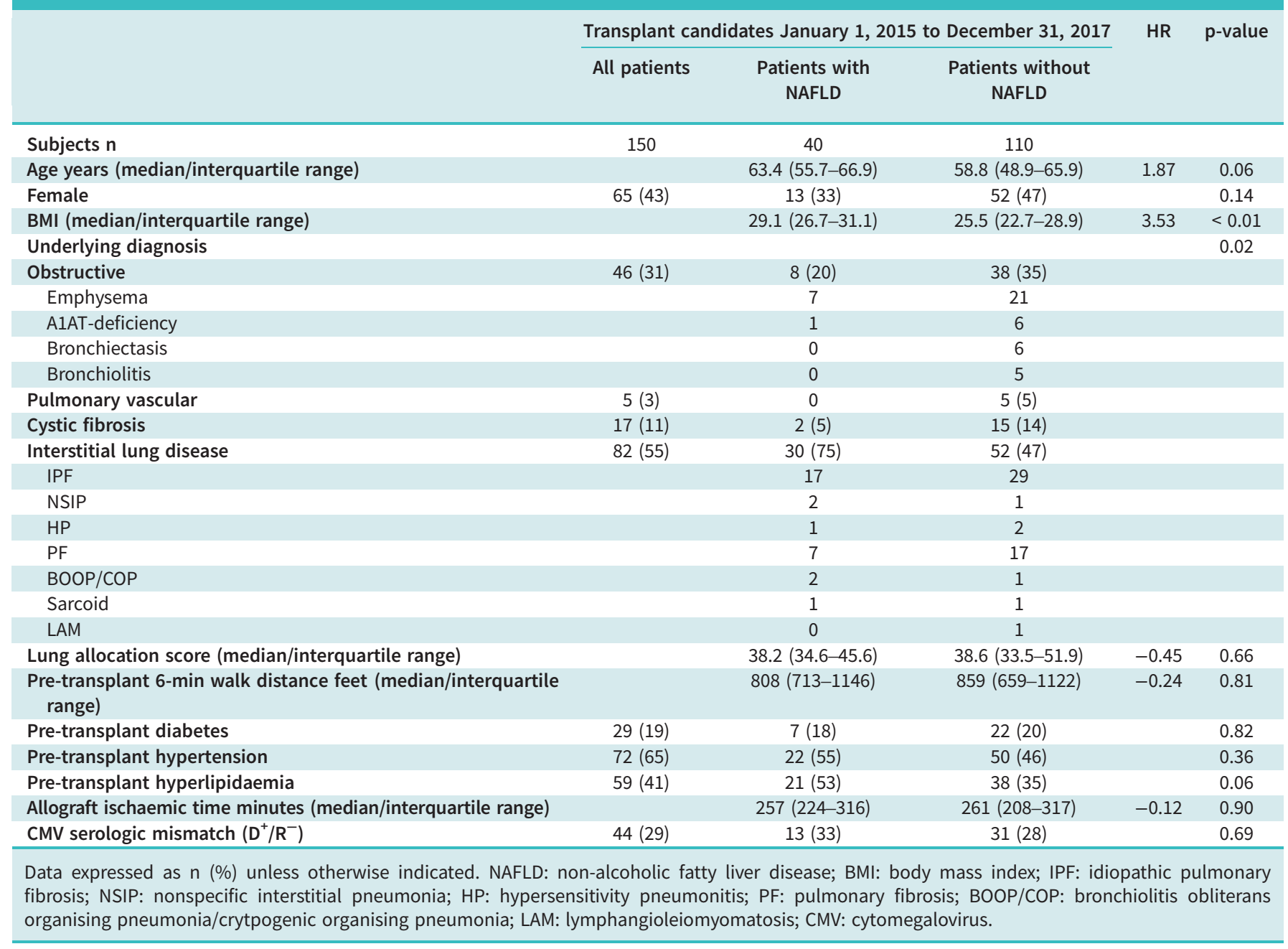

\section{Discussion}

Our results show that lung allograft recipients with NAFLD with mild-moderate fibrosis have non-inferior 1-year post-transplant outcomes, compared to patients without NAFLD. NAFLD does not seem to contribute to increased morbidity, despite the association with significant comorbidities in the general population, including hypertension, diabetes, atrial fibrillation and chronic kidney disease. This finding is important given the wide prevalence of hepatic steatosis and fibrosis. It may be that proinflammatory signalling that contributes to the pathogenesis of NAFLD is less significant than the effects of alloimmunity and immunosuppression.

The correlation between NAFLD and index hospital-free days in this study cannot be readily explained. We do not believe that NAFLD would confer any benefits related to perioperative recovery post-transplant. We demonstrate that NAFLD is not associated with inferior outcomes post-transplant.

Our study has several strengths. One is the generalizability of our population to that of most lung transplant centres, based on similar baseline demographics reported in other studies and registries [38]. Another strength is the multimodal approach to characterising fatty liver disease in our patient population. This is particularly important given the lack of an agreed upon gold standard for diagnosing NAFLD.

Our study also has several limitations. First, is the retrospective, single-centre design. Second, our study suffers from a lack of global consensus for diagnosing NAFLD. We did utilise abdominal ultrasound testing in all patients, which is the currently accepted initial mode of evaluation, though this may have missed detection of mild disease in some patients [26]. This is why we utilised other testing modalities for liver disease in patients with risk factors. Advanced testing was guided by hepatology expertise and patient 
TABLE 3 One-year outcomes in lung transplant recipients with NAFLD

Outcome (within 1 year post-transplantation unless otherwise specified)
Patients with NAFLD
Patients without p-value NAFLD

\begin{tabular}{|c|c|c|c|}
\hline ICU-free days within 10 days post-transplant (median, IQR) & $5.0(2.0-6.0)$ & $3.0(0-6.0)$ & 0.27 \\
\hline Hospital-free days within 30 days post-transplant (median, IQR) & $16.0(10.5-19.5)$ & $12.0(0-18.0)$ & 0.03 \\
\hline \multicolumn{4}{|l|}{ Transfusion (within 3 days post-transplant) } \\
\hline Packed red blood cells $>2$ units & $11 / 40(28)$ & $47 / 110(43)$ & 0.13 \\
\hline Fresh frozen plasma $>2$ units & $17 / 40(43)$ & $39 / 110(36)$ & 0.45 \\
\hline Platelets (any) & $21 / 40(53)$ & $46 / 110(42)$ & 0.27 \\
\hline Cryoprecipitate (any) & $10 / 40(25)$ & $19 / 110(17)$ & 0.35 \\
\hline Atrial fibrillation during index hospitalisation & $21 / 40(53)$ & $46 / 108(42)$ & 0.35 \\
\hline Delirium during index hospitalisation & $16 / 40(40)$ & $55 / 108(51)$ & 0.27 \\
\hline $\begin{array}{l}\text { Daily tacrolimus dose required to maintain a goal trough of } 8-12 \mathrm{ng} / \mathrm{mL} \text { prior to index } \\
\text { hospitalisation discharge } \mathrm{mg} \text { (median, IQR) }\end{array}$ & $6.0(4.0-8.5)$ & $8.0(5.0-10.0)$ & 0.66 \\
\hline Venous thromboembolism within initial 12 months ${ }^{\#}$ & $16 / 38(42)$ & $44 / 103(43)$ & 1.00 \\
\hline Acute cellular rejection event (any grade, within initial 12 months) ${ }^{\#}$ & $18 / 38(47)$ & $41 / 10240)$ & 0.45 \\
\hline Need for insulin at time of discharge from index hospitalisation & $29 / 40(73)$ & $70 / 104(67)$ & 0.77 \\
\hline Need for insulin within initial 12 months ${ }^{\#}$ & $16 / 38(42)$ & $31 / 102(30)$ & 0.23 \\
\hline$\%$ decline of pre-transplant glomerular filtration rate (CKD-EPI) at 12 months ${ }^{\#}$ & $48(33-57)$ & $58(42-68)$ & 0.03 \\
\hline Unplanned re-hospitalisations within initial 12 months post-transplant ${ }^{\#}$ & $2.0(1.0-3.0)$ & $2.0(1.0-3.0)$ & 0.43 \\
\hline CMV viraemia (viral load $>137$ copies) incidence within initial 12 months post-transplant & $6 / 40(15)$ & $19 / 110(17)$ & 0.74 \\
\hline Hospitalisation for infection during initial 12 months post-transplant ${ }^{\#}$ & $20 / 38(53)$ & $43 / 102(42)$ & 0.34 \\
\hline
\end{tabular}

Data expressed as $n$ (\%) unless otherwise indicated. NAFLD: non-alcoholic fatty liver disease; ICU: intensive care unit; CKD-EPI: Chronic Kidney Disease Epidemiology Collaboration; CMV: cytomegalovirus. \#: for those surviving to the given outcome.

clinical status and was not intended to be formulaic. Rather a pragmatic, multimodal approach to excluding severe liver disease was adopted, given the clinical demands of scheduling FibroScan studies in sick patients and the need for timely lung transplant evaluation. Another limitation was the study size. While we did have sufficient power to assess differences in hospital-free days, we did not have sufficient power to assess other pertinent outcomes such as decline in renal function or incidence of acute cellular rejection. Finally, this analysis is limited to assessing outcomes of patients with NAFLD 1 year following transplantation; a longer follow-up period is likely needed to identify the negative impacts of NAFLD on extrahepatic comorbidities in lung transplant recipients, such as diabetes and cardiovascular disease.

TABLE 4 Multivariate analysis of 1-year outcomes in transplant recipients with non-alcoholic fatty liver disease (NAFLD) (adjusting for age, BMI, lung allocation score disease diagnosis group and pre-transplant hyperlipidaemia)

\begin{tabular}{|c|c|c|}
\hline Outcome (within 1-year post-transplantation unless otherwise specified) & Odds ratio ${ }^{\#}(95 \% \mathrm{Cl})$ & p-value \\
\hline ICU-free days within 10 days post-transplant & $0.71(-0.39-1.79)$ & 0.21 \\
\hline Hospital-free days within 30 days post-transplant & $3.34(0.50-6.19)$ & 0.02 \\
\hline \multicolumn{3}{|l|}{ Transfusion (within 3 days post-transplant) } \\
\hline Packed red blood cells $>2$ units & $0.76(0.32-1.79)$ & 0.53 \\
\hline Fresh frozen plasma $>2$ units & $1.63(0.72-3.68)$ & 0.24 \\
\hline Platelets (any) & $2.23(0.98-5.08)$ & 0.06 \\
\hline Cryoprecipitate (any) & $1.81(0.71-4.58)$ & 0.21 \\
\hline Atrial fibrillation during index hospitalisation & $1.33(0.58-3.02)$ & 0.50 \\
\hline Delirium during index hospitalisation & $0.59(0.27-1.33)$ & 0.20 \\
\hline Venous thromboembolism within initial 12 months ${ }^{\#}$ & $1.06(0.48-2.35)$ & 0.88 \\
\hline Acute cellular rejection (any grade within initial 12 months) & $1.20(0.55-2.66)$ & 0.65 \\
\hline Need for insulin (on index hospitalisation discharge) & $1.23(0.51-2.96)$ & 0.64 \\
\hline Need for insulin within initial 12 months & $1.67(0.72-3.88)$ & 0.23 \\
\hline Decline of pre-transplant glomerular filtration rate (CKD-EPI) & $-0.07(-0.15-0.01)$ & 0.07 \\
\hline$\geqslant 2$ unplanned re-hospitalisations within initial 12 months & $1.18(0.49-2.85)$ & 0.72 \\
\hline Hospitalisation for infection within initial 12 months & $1.44(0.66-3.14)$ & 0.36 \\
\hline
\end{tabular}


Notably, we chose to exclude patients with F3-F4 liver disease, a priori, to minimise perceived risks to this sub-population. Our current study provides justification to consider additional clinical studies assessing the safety of performing lung transplantation in patients with more advanced liver disease.

To our knowledge this is the first study to report on outcomes of lung transplant recipients with NAFLD with mild-moderate fibrosis. Based upon our findings, such patients should not be excluded from lung transplantation based upon their liver disease alone.

Conflict of interest: None declared.

\section{References}

1 Weill D, Benden C, Corris PA, et al. A consensus document for the selection of lung transplant candidates: 2014 - an update from the Pulmonary Transplantation Council of the International Society for Heart and Lung Transplantation. J Heart Lung Transplant 2015; 34: 1-15.

2 Byrne CD, Targher G. NAFLD: a multisystem disease. J Hepatol 2015; 62: Suppl. 1, S47-S64.

3 Papić N, Jelovčić F, Karlović M, et al. Nonalcoholic fatty liver disease as a risk factor for Clostridioides difficile infection. Eur J Clin Microbiol Infect Dis 2020; 39: 569-574.

4 Jiang $\mathrm{T}$, Chen X, Xia C, et al. Association between Helicobacter pylori infection and non-alcoholic fatty liver disease in North Chinese: a cross-sectional study. Sci Rep 2019; 9: 4874,

5 Di Minno MN, Tufano A, Rusolillo A, et al. High prevalence of nonalcoholic fatty liver in patients with idiopathic venous thromboembolism. World J Gastroenterol 2010; 16: 6119-6122.

6 Sung KC, Seo DC, Lee SJ, et al. Non alcoholic fatty liver disease and risk of incident diabetes in subjects who are not obese. Nutr Metab Cardiovasc Dis 2019; 29: 489-495.

7 Targher G, Mantovani A, Pichiri I, et al. Nonalcoholic fatty liver disease is independently associated with an increased incidence of chronic kidney disease in patients with type 1 diabetes. Diabetes Care 2014; 37: 1729-1736.

8 Zoncu R, Efeyan A, Sabatini DM. mTOR: from growth signal integration to cancer, diabetes and ageing. Nat Rev Mol Cell Biol 2011; 12: 21-35.

9 Jang HR, Kang D, Sinn DH, et al. Nonalcoholic fatty liver disease accelerates kidney function decline in patients with chronic kidney disease: a cohort study. Sci Rep 2018; 8: 4718.

10 Kok HM, Falke LL, Goldschmeding R, et al. Targeting CTGF, EGF and PDGF pathways to prevent progression of kidney disease. Nat Rev Nephrol 2014; 10: 700-711.

11 Villanova N, Moscatiello S, Ramilli S, et al. Endothelial dysfunction and cardiovascular risk profile in nonalcoholic fatty liver disease. Hepatology 2005; 42: 473-480.

12 Tripodi A, Fracanzani AL, Chantarangkul V, et al. Procoagulant imbalance in patients with non-alcoholic fatty liver disease. J Hepatol 2017; 66: 248-250.

13 Bhanji RA, Narayanan P, Allen AM, et al. Sarcopenia in hiding: the risk and consequence of underestimating muscle dysfunction in nonalcoholic steatohepatitis. Hepatology 2017; 66: 2055-2065.

14 Jenssen T, Hartmann A. Post-transplant diabetes mellitus in patients with solid organ transplants. Nat Rev Endocrinol 2019; 15: 172-188.

15 Saad M, Elgendy IY, Mentias A, et al. Incidence, predictors, and outcomes of early atrial arrhythmias after lung transplant: a systematic review and meta-analysis. JACC Clin Electrophysiol 2017; 3: 718-726.

16 Silverborn M, Jeppsson A, Mårtensson G, et al. New-onset cardiovascular risk factors in lung transplant recipients. J Heart Lung Transplant 2005; 24: 1536-1543.

17 Marchetti P. New-onset diabetes after transplantation. J Heart Lung Transplant 2004; 23: Suppl. 5, S194-S201. doi:

18 Moniodis A, Townsend K, Rabin A, et al. Comparison of extracorporeal photopheresis and alemtuzumab for the treatment of chronic lung allograft dysfunction. J Heart Lung Transplant 2018; 37: 340-348.

19 Poynard T, Vergniol J, Ngo Y, et al. Staging chronic hepatitis C in seven categories using fibrosis biomarker (FibroTest ${ }^{\mathrm{TM}}$ ) and transient elastography (FibroScan ${ }^{\circledR}$ ). J Hepatol 2014; 60: 706-714.

20 Byrne CD, Patel J, Scorletti E, et al. Tests for diagnosing and monitoring non-alcoholic fatty liver disease in adults. BMJ 2018; 362: k2734.

21 Sharma S, Khalili K, Nguyen GC. Non-invasive diagnosis of advanced fibrosis and cirrhosis. World $J$ Gastroenterol 2014; 20: 16820-16830.

22 Bedossa P, Poynard T. An algorithm for the grading of activity in chronic hepatitis C. The METAVIR Cooperative Study Group. Hepatology 1996; 24: 289-293.

23 Ferraioli G, Tinelli C, Dal Bello B, et al. Accuracy of real-time shear wave elastography for assessing liver fibrosis in chronic hepatitis C: a pilot study. Hepatology 2012; 56: 2125-2133.

24 Kumar R, Rastogi A, Sharma MK, et al. Liver stiffness measurements in patients with different stages of nonalcoholic fatty liver disease: diagnostic performance and clinicopathological correlation. Dig Dis Sci 2013; 58: $265-274$. 
Shaheen AA, Wan AF, Myers RP. FibroTest and FibroScan for the prediction of hepatitis C-related fibrosis: a systematic review of diagnostic test accuracy. Am J Gastroenterol 2007; 102: 2589-2600.

Yoneda M, Fujita K, Inamori M, et al. Transient elastography in patients with non-alcoholic fatty liver disease (NAFLD). Gut 2007; 56: 1330-1331.

Naveau S, Raynard B, Ratziu V, et al. Biomarkers for the prediction of liver fibrosis in patients with chronic alcoholic liver disease. Clin Gastroenterol Hepatol 2005; 3: 167-174.

Ratziu V, Massard J, Charlotte F, et al. Diagnostic value of biochemical markers (FibroTest-FibroSURE) for the prediction of liver fibrosis in patients with non-alcoholic fatty liver disease. BMC Gastroenterol 2006; 6: 6 .

Naveau S, Gaudé G, Asnacios A, et al. Diagnostic and prognostic values of noninvasive biomarkers of fibrosis in patients with alcoholic liver disease. Hepatology 2009; 49: 97-105.

Lubner MG, Pickhardt PJ. Multidetector computed tomography for assessment of hepatic fibrosis. Clin Liver Dis (Hoboken) 2018; 11: 156-161.

1 Pickhardt PJ, Malecki K, Kloke J, et al. Accuracy of liver surface nodularity quantification on MDCT as a noninvasive biomarker for staging hepatic fibrosis. AJR Am J Roentgenol 2016; 207: 1194-1199.

Bohte $\mathrm{AE}$, van Werven JR, Bipat $\mathrm{S}$, et al. The diagnostic accuracy of US, CT, MRI and $1 \mathrm{H}-\mathrm{MRS}$ for the evaluation of hepatic steatosis compared with liver biopsy: a meta-analysis. Eur Radiol 2011; 21: 87-97.

Venkatesh SK, Yin M, Takahashi N, et al. Non-invasive detection of liver fibrosis: MR imaging features vs. MR elastography. Abdom Imaging 2015; 40: 766-775.

Gusmao-Flores D, Figueira Salluh JI, Chalhub RA, et al. The confusion assessment method for the intensive care unit (CAM-ICU) and intensive care delirium screening checklist (ICDSC) for the diagnosis of delirium: a systematic review and meta-analysis of clinical studies. Crit Care 2012; 16: R115.

35 Stewart S, Fishbein MC, Snell Gl, et al. Revision of the 1996 working formulation for the standardization of nomenclature in the diagnosis of lung rejection. J Heart Lung Transplant 2007; 26: 1229-1242.

6 Levey AS, Stevens LA. Estimating GFR using the CKD Epidemiology Collaboration (CKD-EPI) creatinine equation: more accurate GFR estimates, lower CKD prevalence estimates, and better risk predictions. Am J Kidney Dis 2010; 55: 622-627.

7 Castera L, Winnock M, Pambrun E, et al. Comparison of transient elastography (FibroScan), FibroTest, APRI and two algorithms combining these non-invasive tests for liver fibrosis staging in HIV/HCV coinfected patients: ANRS CO13 HEPAVIH and FIBROSTIC collaboration. HIV Med 2014; 15: 30-39.

Chambers DC, Cherikh WS, Goldfarb SB, et al. The International Thoracic Organ Transplant Registry of the International Society for Heart and Lung Transplantation: Thirty-fifth adult lung and heart-lung transplant report-2018; Focus theme: Multiorgan Transplantation. J Heart Lung Transplant 2018; 37: 1169-1183. 